\title{
CORPORATE GOVERNANCE KODEX
}

\section{Chancen für \\ Non-Profit- \\ Organisationen}

\author{
Robert Bachert
}

\begin{abstract}
Zunehmend erkennen Non-Profit-Organisationen die Vorteile einer standardisierten Unternehmensführung. Die haupt- und ehrenamtlichen Führungs- und Aufsichtsgremien sind den vielfältigen Anforderungen eines sich schnell wandelnden Umfeldes institutionell und instrumentell oftmals nicht gewachsen. Kompetenzen, Zuständigkeiten, Rollen und Aufgaben sind in der Organisation nicht definiert. Die Einführung eines Corporate Governance Kodex kann dem abhelfen.
\end{abstract}

Die Vermeidung von Unternehmenskrisen in Wirtschaft, Industrie und Sozialbranche erfordern die Einhaltung von Standards der Unternehmensleitung - und eine Aufsicht. Diese finden sich beispielsweise im Deutschen Corporate Governance Kodex wieder.

Die in jüngster Zeit entwickelten und verabschiedeten Kodices der Corporate Governance für Non- ProfitOrganisationen, aber auch der Deutsche Corporate Governance Kodex stellen eine Art Rahmenkonzept für eine gute Unternehmensführung dar. Zwei Beispiele für Kodizes in der NonProfit-Szene sind der »Corporate Governance Kodex des Diakonischen Werks der EKD « (Stand Oktober 2005) und der »Corporate Governance Kodex des Diakonischen Werks Württemberg e. V.« (Stand November 2005).

\section{Was bedeutet Corporate Gover- nance?}

»Der feststehende Ausdruck >Corporate Governance $<$ leitet sich ab aus `Corporate $<$, das man mit körperschaftlich übersetzen kann und >Governance $<$, das seinen Ursprung im Lateinischen gubernare in der Bedeutung von das Steuerruder führen, lenken, leiten hat.« (Solidaris, 2003, Seite 7). »In diesem Sinne könnte man Corporate Governance definieren als ein System mit dem Unternehmen geführt und kontrolliert werden. Die Kernaufgabe der Corporate Governance besteht darin, die Führung und Kontrolle so zu gestalten, dass die langfristige Existenzsicherung der Organisation gewährleistet wird und dass es zu einem Interessenausgleich zwischen allen internen und externen Anspruchsgruppen kommt.«(Bachert, 2005, Seite 197). Der Deutsche Corporate Governance Kodes (DCGK) ist 2002 verabschiedet und seit dem regelmäßig an aktuelle Entwicklungen angepasst worden.

Eine Grundlage des Deutschen Corporate Governance Kodex bildet das Gesetz zur Kontrolle und Transparenz im Unternehmensbereich (KonTraG). Sein Ziel ist, die Kontrolle und Transparenz bei Unternehmen zu erhöhen.

Die gesetzliche Umsetzung geschah durch Änderungen des Aktien- und Handelsgesetzes (vgl. Solidaris, 2003, Seite 7). »Darauf aufbauend wurden im Jahr 2002 mit dem Deutschen Corporate Governance Kodex (DCGK) zusätzliche Empfehlungen erarbeitet, die im selben Jahr durch das Gesetz zur weiteren Reform des Aktien- und Bilanzrechts, zu Transparenz und Pu-

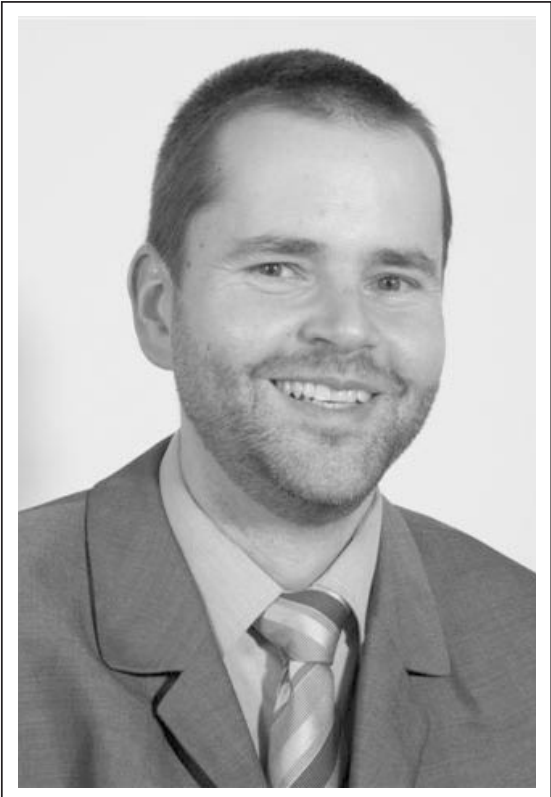

Robert Bachert ist Diplom-Sozialpädagoge (BA) und DiplomBetriebswirt (BA). Seit über fünf Jahren ist er Geschäftsführer der ZSU GmbH (Zentraler Buchungsstelle Sozialer Unternehmen) und seit Ende 2001 zusätzlich Abteilungsleiter der Wirtschaftsberatung im Diakonischen Werk Württemberg. Von 1999 bis 2001 war er Seniorberater und Trainer bei der $B S U$ (Wirtschaftsberatungsgesellschaft Sozialer Unternehmen).

E-Mail bachert-rom@t-online.de

blizitätsgesetz - kurz Transparenz und Publizitätsgesetz (TransPuG) genannt - weitestgehend umgesetzt worden sind. (...) Dieser liefert über die gesetzlichen Vorschriften zur Leitung und Überwachung deutscher börsennotierter Aktiengesellschaften (AG) hinaus konkrete Handlungsempfehlungen bzw. Standards für eine gute und verantwortliche Führung durch die Vorstände und Aufsichtsräte und deren Zusammenarbeit mit der Hauptversammlung bzw. den Gesellschaftern (Aktionären) « (Solidaris, 2003, Seite 7).

Es stellt sich die Frage, inwieweit der Gedanke der Corporate Governance auf die Sozialbranche übertragbar ist. Dazu kann gesagt werden, dass beispielsweise die Anwendung des Unternehmensbegriffs auf die gemeinnützigen Organisationen in der einschlägigen Fachliteratur legitimiert wird. Das KonTraG zielt zwar in 


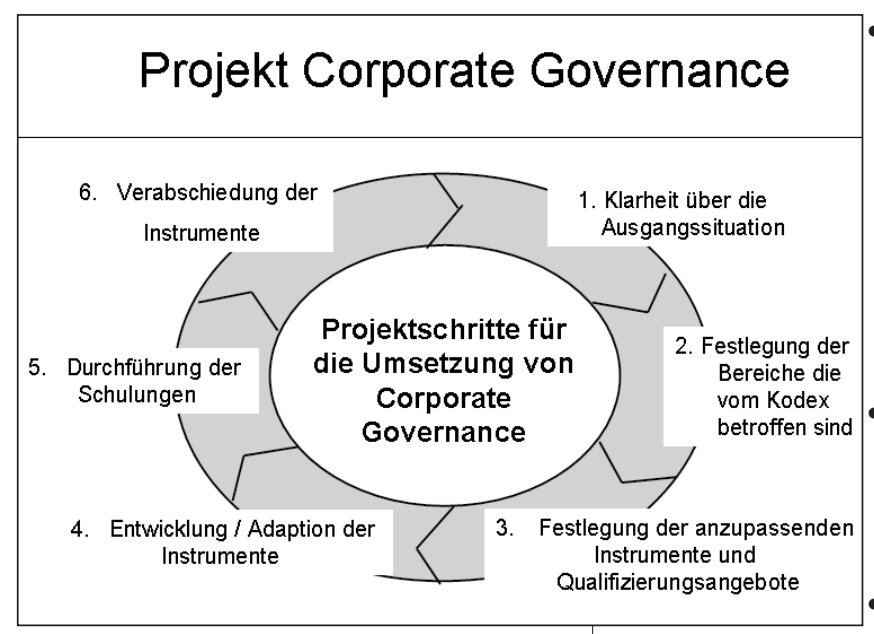

Grafik 1: Ein möglicher Phasenplan zeigt die Schritte zur Einführung eines Kodexes.

erster Linie auf börsennotierte Aktiengesellschaften $\mathrm{ab}$, dennoch hat es auch Auswirkungen auf gemeinnützige Organisationen. Dies gilt insbesondere für diejenigen, die in der Rechtsform der GmbH geführt werden. Selbst wenn bestimmte Regelungen nicht gesetzlich zwingend sind, geht der Gesetzgeber von einer Ausstrahlungswirkung aus (vgl. Schuhen, 2003, Seite 95). »Unternehmen gleich welcher Größe, Rechtsform oder Ertragskraft, die sich einer guten Corporate Governance verschließen, werden auf lange Sicht gegenüber Mitbewerbern weniger erfolgreich sein, die den DCGK umgesetzt haben.«(Pfitzer/Oser, 2003, Seite 8)

\section{Chancen der Anwendung}

Non-Profit-Organisationen stehen der Anwendung eine Corporate Governance Kodexes zum Teil kritisch gegenüber. Es wird dabei die Frage eine Rolle spielen, ob es sich lohnt, einen Kodex anzuwenden und dies auch einer breiten Öffentlichkeit gegenüber bekannt zu geben. Gründe und Vorteile der Anwendung stellen sich wie folgt dar (vgl. dazu Bachert, 2005, Seiten 199 f.):

Welche Gründe gibt es für die Anwendung?

Gute Gründe für die Anwendung eines Corporate Governance Kodexes in der Non-Profit-Organisation lassen sich über die folgenden Stichworte charakterisieren:

- Die Entwicklung und Kommunikation des Zielsystems der jeweiligen Non-Profit-Organisation wird geleistet.
- Die Festlegung der Grundsätze in Bezug auf die Aufbau- und Ablauforganisation für das System der Unternehmensführung und dessen Kontrolle wird erledigt.

- Die transparente Trennung zwischen Aufsicht und Leitung wird befördert.

- Die transparente Entwicklung, Dokumentation und Anwendung von betriebswirtschaftlichen Instrumenten in Bezug auf Risikomanagement, Controlling und Aufsicht und Führung zur Erreichung einer guten und verantwortungsvollen Unternehmensführung wird erreicht.

- Bestrebungen um eine höhere Qualität und optimale Qualifikation der Aufsichtsgremien (z. B. Effizienzprüfung der Sitzungen der Aufsichtsgremien) werden unternommen.

Welche Vorteile sind bei der Anwendung eines Corporate Governance Kodexes erkennbar?

Vorteile, die sich daraus ergeben können, sind:

- Führungskräfte und Mitglieder der Aufsichtsgremien sind zur Erledigung ihrer Aufgaben optimal qualifiziert.

- Führungskräfte und Aufsichtsgremien sind über die Themen der Corporate Governance sensibilisiert und haben ein Bewusstsein darüber, welche Rechte und Pflichten sie haben.

- Vertrauen der Öffentlichkeit in die Managementkompetenz der entsprechenden Non-Profit-Organisa tion wird gestärkt.

- Wesentliche Wettbewerbsvorteile bei der Spendenakquisition werden generiert.

- Insolvenzen werden vermieden.

- Belegungsvorteile entstehen.

- Liquiditätsvorteile ergeben sich.

- Ein besseres Rating nach Basel II durch die Banken erfolgt.

- Unterschiedliche betriebswirtschaftliche Instrumente kommen im Sinne einer Toolbox optimiert zum Einsatz.

\section{Projektfahrplan zur Umsetzung}

Ein Projekt zur Umsetzung eines Kodexes, welcher die Entwicklung der benötigten betriebswirtschaftlichen Instrumente einbezieht, wird in unterschiedlichen Phasen ablaufen. Die Grafik 1 zeigt einen denkbaren Phasenplan.

\section{Phase 1: Klarheit über die Aus- gangssituation}

Die erste Phase dient dazu, eine Klärung der Ausgangssituation herbeizuführen. Dabei kommt es vor allem darauf an, alle Gesichtspunkte im Sinne von Chancen und Risiken transparent aufzulisten. Ferner muss Klarheit darüber bestehen, dass die Einführung eines Corporate Governance Kodexes immer auch den Einsatz von Zeit und Geld erfordert. Die nachfolgende Checkliste gibt einen Überblick der grundlegenden Fragen, die zu klären sind:

- Sind wir verpflichtet, einen Corporate Governance Kodex einzuführen?

- Wenn wir nicht dazu verpflichtet sind: Ließen sich die Belange eines Kodexes auch auf anderem Weg verwirklichen?

- Haben wir bereits Gesichtspunkte, die der uns betreffende Kodex aufgreift, in unserer Organisation verwirklicht (z. B. Regelungen im Qualitätsmanagement, Instrumente im Controlling und Risikomanagement, in der Satzung oder Geschäftsordnung)?

- Wenn ja, in welchen Bereichen sind Regelungen und Standards bereits jetzt mit dem Kodex kompatibel und wo gibt es Handlungsbedarf?

- Sind unsere Führungskräfte im Sinne des Kodexes ausreichend für Ihre Führungsaufgaben qualifiziert und werden sie den Anforderungen des Kodexes gerecht?

- Sind die Mitglieder unserer Aufsichtsgremien im Sinne des Kodexes ausreichend für Ihre Führungsaufgaben qualifiziert und werden sie den Anforderungen Kodexes gerecht?

- Stehen ausreichend personelle und finanzielle Ressourcen für die Implementierung und Umsetzung des Kodexes zur Verfügung?

- Ist der Wille vorhanden, die nötigen finanziellen Mittel zur Verfügung zu stellen, um die Instrumentenentwicklung und die 


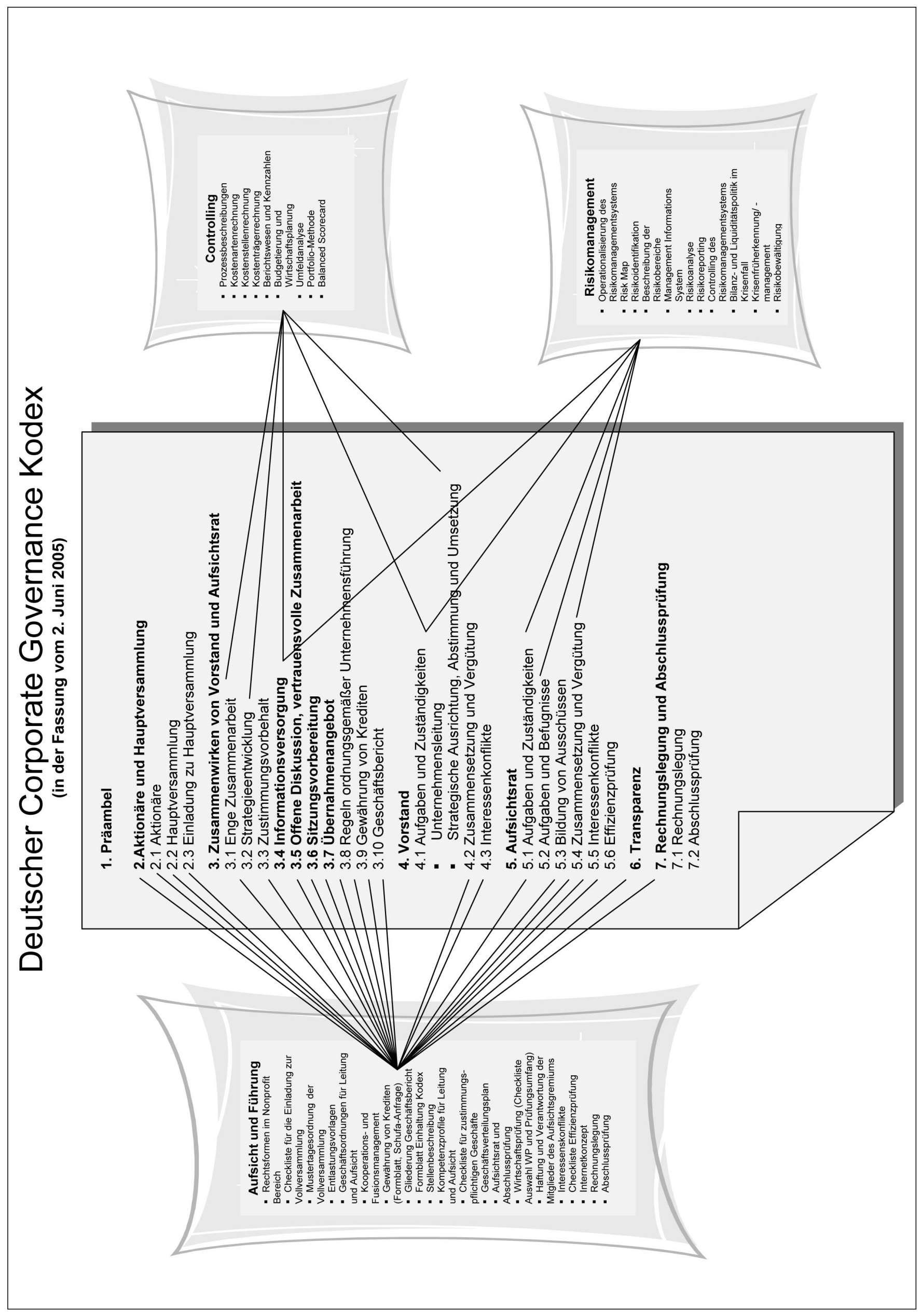


Qualifizierung der Personen zu gewährleisten?

- Sind die dokumentierten Regelungen in unserer Organisation, die den Kodex betreffen, mit dem entsprechenden Kodex konform (z. B. Qualifikation der Aufsichtsräte, Altersbegrenzungen der Führungsund Aufsichtsgremien, Berichtsbestandteile und Berichtspflichten des Vorstands gegenüber den Aufsichtsgremien)?

Phase 2: Festlegung der Bereiche, die vom Kodex betroffen sind

In Phase zwei wird zunächst ein internes Projektteam ins Leben gerufen, welches zunächst ermittelt wo bereits Gesichtspunkte in der Organisation im Sinne des Kodexes bearbeitet werden und wo es Handlungsbedarf gibt. Eine mögliche Einteilung in Bereiche kann sich wie folgt gestalten: Aufsicht und Führung; Controlling und Risikomanagement. kommuniziert. Zeitgleich dazu wird die Phase 5 durchgeführt.

\section{Phase 5: Durchführung der Schu- lungen}

Die Führungskräfte und Aufsichtsgremien werden zu den Inhalten des Kodexes geschult. Stichworte dabei sind die Sensibilisierung und die Bewusstseinsbildung über die Aufgaben dieser Funktionen und der damit einhergehenden Pflichten. Ein Ziel der Schulungen kann neben der Kompetenzerweiterung auch eine Verhaltensänderung bei den verantwortlichen Aufsichts- und

Führungsgremien darstellen.

\section{Phase 6: Verabschiedung der In-} strumente, Qualifizierung

Die Instrumente zur Verwirklichung guter Corporate Governance werden verabschiedet und beispielsweise im

\section{" Jede Non-Profit-Organisation sollte prüfen, inwieweit sie Standards der Corporate Governance erfüllt «}

\section{Phase 3: Festlegung der Instrumente und Qualifizierungs- angebote}

Die Regelungen der Corporate Governance können mit Hilfe von Instrumenten der Aufsicht und Führung, des Controllings und des Risikomanagements umgesetzt werden. In dieser Phase geht es darum diejenigen auszuwählen oder zu entwickeln, die noch nicht zum Einsatz kommen. Die Grafik 2 (Seite 27) stellt die Gliederung des Deutschen Corporate Governance Kodexes dar und listet unter den genannten Rubriken mögliche Instrumente auf. Daneben ist der Qualifizierungsbedarf der Führungskräfte und Aufsichtsgremien festzustellen.

\section{Phase 4: Adaption und Entwick- lung der Instrumente}

Die ausgewählten Instrumente werden an die Erfordernisse der Organisation adaptiert. Gegebenenfalls werden neue Instrumente entwickelt und
Qualitätsmanagement-Handbuch dokumentiert und zur Verfügung gestellt. Die Instrumente werden im Sinne einer Toolbox angewendet.

\section{Fazit}

Unabhängig von ihren jeweiligen Rechtsformen sollten Non-Profit-Organisation prüfen, inwieweit sie bereits jetzt die Standards der Corporate Governance umsetzen. Sollte eine Organisation feststellen, dass sowohl Instrumente, als auch Regelungen der Ablauf- und Aufbau-Organisation nicht den Anforderungen entsprechen, ist ein Projekt zur Implementierung und Umsetzung der Corporate Governance anzugehen.

Im Gegensatz zu Aktiengesellschaften ist der Non-Profit-Sektor nicht nur den Anlegern gegenüber verantwortlich, sondern der Kreis der vom Handeln der Sozialbranche Betroffenen, ist weiter zu fassen. Hier geht es um Mitarbeitende, Kunden, Kostenträger, staatliche Institutionen, Ärzte,
Versicherungsträger, Angehörige und Banken.

Letztlich sollten alle Non-Profit-Organisationen mindestens ähnlich hohe Anforderungen an sich selber haben, als dies für Betriebe der Industrie und Wirtschaft gilt.

\section{Literatur}

Robert Bachert: Corporate Governance in der Non-Profit-Organisation, 2006, Haufe Verlag.

Robert Bachert, in Ruter, Sahr, Waldersee, Public Corporate Governance, 2005, Gabler-Verlag.

Norbert Pfitzner, Peter Oser ( 2003 ): Deutscher Corporate Governance Kodex, Schäffer Poeschel Verlag, Stuttgart. 